\title{
Polymer-Based Enzyme-Linked Immunosorbent Assay
}

\author{
Long Liu ${ }^{1}$, Aihong Zhang ${ }^{2}$ and Xinghe Wang ${ }^{1 *}$ \\ ${ }^{1}$ Phase I Clinical Trial Center, Beijing, China \\ ${ }^{2}$ Nuclear biochemical emergency technical support center, Beijing, China \\ *Corresponding author: Xinghe Wang, Phase I Clinical Trial Center, Beijing, China 100038
}

\begin{tabular}{|c|}
\hline ARTICLE INFO \\
\hline Received: 崫 January 08, 2020 \\
\hline Published: 㗀 January 21, 2020 \\
\hline Citation: Long Liu, Aihong Zhang, Xinghe \\
\hline $\begin{array}{l}\text { Wang. Polymer-Based Enzyme-Linked } \\
\text { Immunosorbent Assay. Biomed J Sci \& Tech }\end{array}$ \\
\hline Res 24(4)-2020. BJSTR. MS.ID.004095. \\
\hline
\end{tabular}

Keywords: Polymer; Enzyme-Linked Immunesorbent Assay

\begin{abstract}
In this study, a new and sensitive enzyme-linked immunosorbent assay (ELISA) was developed by introducing a polymer as a reaction carrier. The results suggest that the newly developed ELISA method is more convenient than the existing paper-based ELISA method and applicable to a wider range of environments. In addition, the sensitivity of the new method is much higher than that of the existing paper-based ELISA method and even higher than that of the traditional ELISA method.
\end{abstract}

\begin{abstract}
Abbreviations: Ab1: Primary Antibody; Ab2: Second Antibody; BSA: Bovine Serum Albumin; EDC: 1-ethyl-3-(3-dimethylaminopropyl) carbodiimide hydrochloride; ELISA: Enzyme-Linked Immunesorbent assay; G3-g-BST ${ }_{3060}$ : third-generation dendrimer-like copolymer; GST: Glutathione S-transferase; HGII: second generation Hoveyda-Grubbs catalyst; HRP: Horseradish peroxidase; LOD: limit of detection; MES: 2-(N-morpholino) ethanesulfonic acid; NgR: Nogo receptor; NHS: N-hydroxysuccinimide; OD: optical density; PBS: Phosphate Buffer Saline; PBST: Phosphate Buffer Saline-Tween 20; PEO: Polyethylene Oxide; RT: Room Temperature; TMB: 3,30,5,50-tetramethyl-benzidine; Tris: tris (hydroxymethyl) aminomethane.
\end{abstract}

\section{Introduction}

ELISA is one of the most effective and popular methods in qualitative and quantitative studies of trace biomarkers or other proteins. This method can amplify the detection signal through the high specific binding between antigen and antibody and the high catalytic rate of enzymes, enabling this detection method to achieve high sensitivity [1,2]. Because ELISA requires expensive equipment and a complex washing process, a new paper-based ELISA method was developed in 2010 [3]. Compared to the traditional ELISA method, this paper-based ELISA method is simpler to use and less expensive, but its sensitivity is not high enough [4-6]. To overcome the above shortcomings, researchers have also introduced graphene oxide sheets [7-9] and gold nanoparticles [10,11] into the traditional ELISA method to amplify the detection signal [12-14]. To some extent, these improvements have solved the problems of low detection signals, high costs and complex processes [15-18]. However, these modified methods are not convenient enough, and their outdoor use is limited [19-21].

In this study, we report a new ELISA method using a polymer as a carrier (polymer-based ELISA, P-ELISA) instead of traditional 96-microzone plates and current 96-microzone paper plates $[22,23]$. Polymers have many advantages over other reaction carriers, such as their abundant functional groups, numerous branched chains and large internal voids $[24,25]$. In addition, the polymer used in this study has no effect on the activity of biological molecules. This honeycomb-like microenvironment is the best place for antibodies to react with proteins, so it can be used as a carrier for a nanoreactor [26-28]. As required, hydrophobic or hydrophilic segments can be introduced into polymer molecules to achieve specific functions. P-ELISA is fast, portable, low cost and reusable. Notably, the new method is an innovation in ELISA carriers, which provides potential for its application in new environments and fields. The polymer used in this study is G3- $g-\mathrm{PEO}_{2900}$ [22]. The molecular weight of the dendrimer-like copolymer peripheral polyethylene oxide (PEO) is as large as $2.16 \times 10^{6}$, with up to 2900 outer PEO arms and an average diameter of $32.8 \mathrm{~nm}$ in solution. The specific preparation process of the polymers is described in the literature. As far as we know, this work is the first time that a polymer used as a carrier is introduced into ELISA to develop a new approach for wider applications. 
To verify the performance of the new method, we selected GST protein and Nogo-66 as models. The polymer is a dendrimer-like structure with a uniform distribution of branches, which increases the probability of binding with antibodies. Because of the narrow molecular weight distribution and the numerous branched chains of the polymer, the formation of a compact shell can greatly reduce the loss of the binding sample and antibody. Each test zone requires only $1 \mu \mathrm{L}$ of solution to fill, and the results can be measured using a desktop scanner, thus greatly reducing equipment costs. In addition, another advantage of using a polymer as a nanocarrier is that the sample added to the test zones is not easy to lose, so the test results are more accurate. The disadvantage of the new method is that the synthesis of polymers is slightly cumbersome. Fortunately, the synthesized polymer can be used many times.

\section{Materials and Methods}

\section{Synthesis of Amphiphilic Dendrimer-like Copolymer (G3-g-PEO ${ }_{2900}$ )}

The specific synthetic route for $\mathrm{G} 3-g-\mathrm{PEO}_{2900}$ is described in detail in the literature [22] and will not be repeated here. The general synthesis process is as follows. First, a living block copolymer (PBSt- $b$-PSLi) and a 3-arm star-like polymer (G1) are synthesized. Second, hydrosilylation of intermediates is carried out
Third, a third-generation dendrimer-like copolymer (G3- $g$-BST ${ }_{3060}$ ) is synthesized. Fourth, amphiphilic dendrimer-like copolymer G3- $g-\mathrm{PEO}_{2900}$ is synthesized. The G3- $g-\mathrm{PEO}_{2900}$ product was placed in a dry vacuum bottle for use. By changing the temperature, the carrier can be converted between activated and inactivated states due to the hydrolysis of the benzyl chloride in the G3- $g-\mathrm{PEO}_{2900}$ nanoreactor.

\section{Design of Polymer Plate}

We used a 24-microzone polymer plate with an array $(3 \times 8)$ of circular test zones for running parallel P-ELISAs (Figure 1). The reason for this design is that it is difficult to deposit the polymer evenly on a large piece of paper. The diameter of each hole was half of the microzone diameter in the traditional 96-microzone plate. Therefore, the size of the test zone of the polymer plates is a quarter of that of the traditional one, which greatly reduces the amount of sample required. Because of the crosslinking of the branched chains, antibodies and proteins can be fully combined, which not only improves the utilization rate of samples but also improves the detection accuracy. Four polymer plates $(8 \times 3)$ can be assembled into a 96 -microzone polymer plate $(8 \times 12)$ to confer compatibility with existing microanalytical infrastructure. Another advantage of this method is that it does not need special equipment to read the results but needs only a mobile phone to take clear pictures.

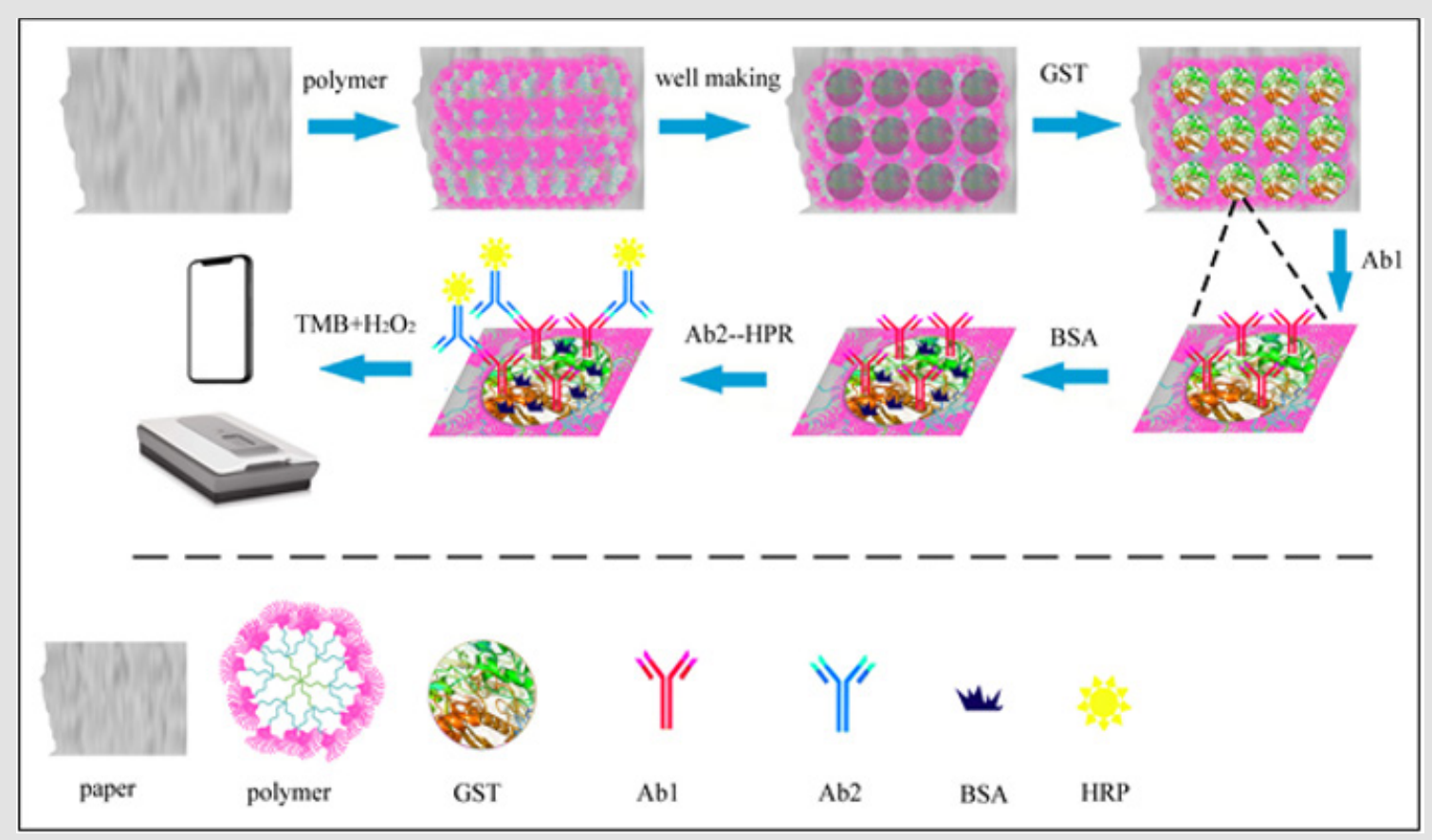

Figure 1: Schematic diagram of the standard P-ELISA procedure.

\section{Preparation of Copolymer Carrier}

To enhance the toughness of the polymer nanocarrier, the polymer was attached to paper in this study. The advantage of this method over other methods is that it is easy to operate and that the porous and branched polymer structure provides better performance. The copolymer, paper and methanol were put into the reactor together and rotated slowly. After 30 minutes, the methanol was removed and dried, and the functionalized paper was preserved for use. Because of affinity, the polymer evenly and firmly deposits on paper. 


\section{Results and Discussion}

The standard procedure of P-ELISA includes the following steps shown in Figure 1:
a) Preparing the copolymer carrier,
b) Fabricating wells,
c) Immobilizing an antigen,
d) Adding the first antibody (Ab1),
e) Blocking the wells to prevent nonspecific adsorption,
f) Washing away the unbound antibody,

g) Adding the second antibody (Ab2) modifying with horseradish peroxidase (HRP),

h) Washing again and

i) Adding the enzyme substrate and measuring the optical density (OD).

To speed up the test, the reacted copolymer carrier can be placed in a ventilated area. If conditions permit, a vacuum pump can be used to accelerate the volatilization of the solution. For specific testing, the process may be adjusted slightly, but the basic operation will not be greatly changed. After the new P-ELISA method was established, the following experiment, which used GST protein as a model, was conducted to examine the performance of the method: Because the gray color intensity is closely related to the quantity of detected substances, the quantity can be obtained according to the gray color intensity. First, the relationship between the gray intensity in the detection area and the sample concentration should be validated. To study the relationship between the gray intensity and the quantity to be measured, the following experiment was carried out: GST protein samples diluted 10 times in turn were detected. The results showed that with increasing sample concentration, the gray color intensity gradually increased; however, there was a linear correlation only within a certain interval, not in all intervals (Figure 2). The color intensity of each test zone was analyzed using ImageJ after scanning the whole microzone plate. The $\mathrm{R}^{2}$ value of the curve fit to the data is 0.993 . In addition, a linear relationship between the concentration dilutions in the range of $1 / 100000$ of $1 / 1000001 / 100$ of raw liquor and the color intensity is basically established.

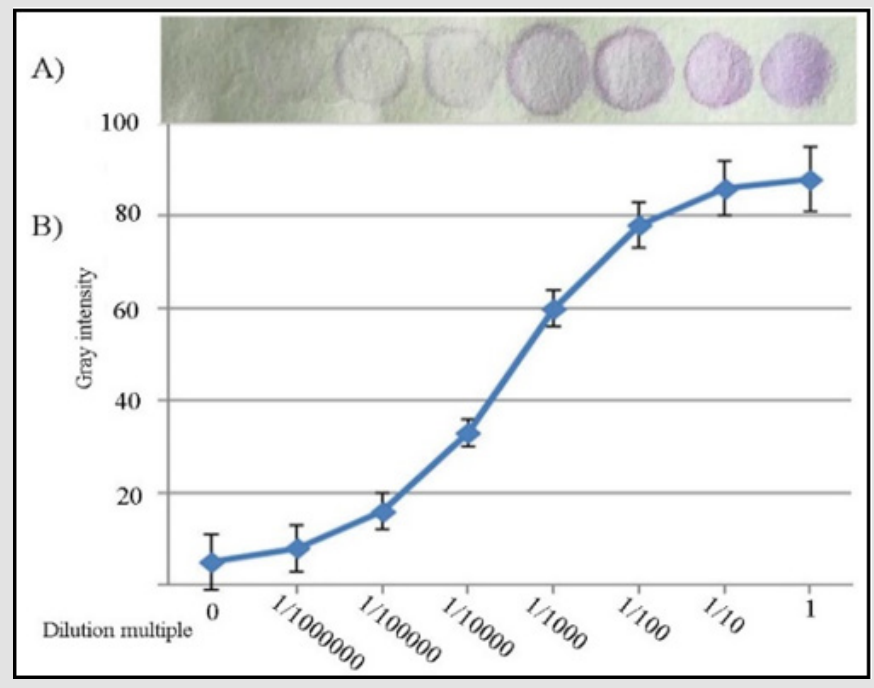

\section{Figure 2:}
A) Images of the P-ELISA results for GST protein with different dilution multiples in each zone.
B) The calibration curve between color intensity and the amount of GST protein in each microzone was determined by the P-ELISA method. Each datapoint is the mean of six replicates $(\mathrm{N}=6)$, and the error bars represent the standard deviations of the measurements.

The curve shows the general relationship between concentration and gray level. However, for different detectors, the relationship curve may be slightly different. To further verify the performance of P-ELISA, we used this method to detect Nogo-66 in human serum samples as well as in control samples (human serum without Nogo66). Nogo was discovered and cloned in recent years and was found to have an inhibitory effect on the central nervous system. Nogo-66 is a 66-amino-acid domain found in the extracellular hydrophilic region of the Nogo protein and is the binding site of the Nogo receptor (NgR). Nogo-66 plays a key role in the Nogo protein, so research on Nogo-66 has attracted much attention in recent years. We examined several dilutions of serum samples from HIV-1positive patients. Specifically, the new P-ELISA plates were coated with Nogo-66 diluted in 30 $\mu \mathrm{L}$ of carbonate buffer at $\mathrm{pH} 9.6$ and incubated at $37^{\circ} \mathrm{C}$ for 10 minutes. After washes with PBST, Nogo66-Ab1 was added and incubated at room temperature (RT) for 10 minutes. After being sealed with 1\% BSA, the plates were incubated at $37^{\circ} \mathrm{C}$ again. After washes with PBST, Ab2-HRP was added, and the plates were incubated at $37^{\circ} \mathrm{C}$. After washing, color was developed using the chromogen/substrate mixture $\mathrm{TMB} / \mathrm{H}_{2} \mathrm{O}_{2}$. 
As shown in Figure 3, the samples in the vertical columns contained the same amount of Nogo-66, and the samples in the horizontal rows represented a 3 -fold dilution series. Nogo-66 was detected in the first three columns using P-ELISA (Figure $3 \mathrm{~A}$ \& 3B), while it was detected only in the first column using the traditional ELISA method (Figure 3C \& 3D). The results show that the P-ELISA method can be used for detection in complex samples such as human serum and that the limit of detection (LOD) of the new method is only one-ninth of that of the traditional method. In other words, the sensitivity of P-ELISA is approximately 9 times higher than that obtained by the traditional ELISA method. The difference in LOD between P-ELISA and traditional ELISA may be a consequence of the high reaction efficiency and low background interference caused by polymer nanocarrier. Table 1 compares the P-ELISA method and the traditional method.

\section{A P-ELISA \\ B control 1 \\ C ELISA \\ D control 2}

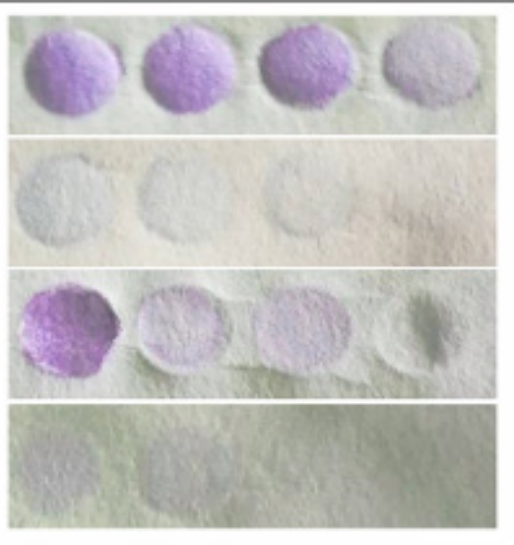

Figure 3: A 3-fold dilution series of Nogo-66 was detected using P-ELISA (A, B) and the traditional ELISA method (C, D). B and D are the control groups detected by the new P-ELISA method and the traditional ELISA method, respectively.

Table 1: Comparison between P-ELISA and conventional ELISA.

\begin{tabular}{|c|c|c|}
\hline \multicolumn{2}{|c|}{ P-ELISA } & Conventional ELISA \\
\hline Carrier & Polymer & Plastic Plate \\
\hline Detection device & Scanner or Smart phone & Plate Reader \\
\hline Sample volume & $2 \mu \mathrm{L}$ & $100 \mu \mathrm{L}$ \\
\hline LOD & $5.6 \mathrm{ng} / \mathrm{mL}$ & $50 \mathrm{ng} / \mathrm{mL}$ \\
\hline Cost & $\$ 0.022 / \mathrm{sample}$ & $\$ 1.802 / \mathrm{sample}$ \\
\hline On-site operation & Yes & No \\
\hline
\end{tabular}

The new P-ELISA method has the following advantages over other detection methods. First, the amount of sample required is less, approximately one-third of that used in the traditional method. Second, the new method took less time. Complete detection took only approximately 60 minutes. Third, the cost is low. The new method does not necessitate expensive equipment, and it is suitable for outdoor use. The reasons for these advantages are as follows: As the area of the test zone is reduced to a quarter of the original area, the amount of sample required is also significantly reduced. Because polymer carriers are nanoscale materials and antibodies react rapidly with antigens, which greatly shorten the whole detection process. The new method does not require additional equipment, and the detection can be performed with only a mobile phone; thus, the cost is greatly reduced. In addition, it is worth mentioning that the nanoreactor can be used repeatedly, and the usefulness of this feature can be explored in future research. Through the previous comparison, we know that compared with the traditional method, the new method has improved sensitivity, cost, detection time, sample quantity, applicability and so on. Because the sensitivity of paper ELISA is lower than that of the traditional ELISA method, the new P-ELISA method has an obvious advantage over paper ELISA in sensitivity.

\section{Conclusions}

In summary, we successfully developed a new and sensitive P-ELISA method based on a polymer as a carrier. Because the new method has a shorter operation process and a lower LOD than those of traditional ELISA, it might enable more extensive application, analysis and environmental monitoring. Because this method is easy to use outdoors and low in price, it can be used for outdoor detection in remote areas or in relatively poor conditions. We believe that the application of polymers in ELISA will provide a useful approach to immunoassays and that this idea will play a greater role in future immunoassays.

\section{Declarations of interest}

none.

\section{References}

1. Chen Z, Wang H, Zhang Z, Chen L (2019) Chemical redox-cycling for improving the sensitivity of colorimetric enzyme-linked immunosorbent assay. Anal Chem 91(2): 1254-1259.

2. Dixit CK, Vashist SK, O'Neill FT, O'Reilly B, Mac Craith BD, et al. (2010) Development of a high sensitivity rapid sandwich ELISA procedure and its comparison with the conventional approach. Anal Chem 82(16): 7049-7052.

3. Cheng CM, Martinez AW, Gong J, Mace CR, Phillips, et al. (2010) Paperbased ELISA. Angew Chem 49(28): 4771-4774.

4. Pang B, Zhao C, Li L, Song X, Xu K, et al. (2018) Development of a lowcost paper-based ELISA method for rapid Escherichia coli 0157: H7 detection. Anal Biochem 542: 58-62.

5. Preechakasedkit P, Siangproh W, Khongchareonporn N Ngamrojanavanich N, Chailapakul O (2018) Development of an automated wax-printed paper-based lateral flow device for alphafetoprotein enzyme-linked immunosorbent assay. Biosens Bioelectron 102: $27-32$.

6. Qi L, Zhang AH, Wang Y, Liu L, Wang XH (2019) Atom transfer radical polymer-modified paper for improvement in protein fixation in paperbased ELISA. BMC Chem 13(1): 110.

7. Zhang X, Ding SN (2017) Graphite paper-based bipolar electrode electrochemiluminescence sensing platform. Biosens Bioelectron 94: 47-55.

8. Liu L, Zhang AH, Wang XH (2019) Ultrasensitive paper-based ELISA by introducing atom transfer radical polymer-modified graphene oxide sheets and gold nanoparticles. Chem Lett 48: 779-782. 
9. Guo C, Zhao X, Zhang W, Bai HH, Qin WJ, et al. (2017) Preparation of polymer brushes grafted graphene oxide by atom transfer radical polymerization as a new support for trypsin immobilization and efficient proteome digestion. Anal Bioanal Chem 409: 4741-4749.

10. Tang F, Zhang S, Zhala G, Huo JR, Tu Y, et al. (2014) Gold-nanoparticlemodified polyvinylidene fluoride membranes used for western blotting with high sensitivity. RSC Adv 4: 31337-31340.

11. Cao QC, Ma C, Bai HH, Li X, Yan H, et al. (2014) Multivalent hydrazidefunctionalized magnetic nanoparticles for glycopeptide enrichment and identification. Analyst 139: 603-609.

12. Lin HJ, Huo JR, Zhang AH, Liu YF, Wang QM, et al. (2012) A sensitive dual signal amplification method for western blotting based on antibodyfunctionalised graphene oxide and gold nanoparticles. Analyst 137: 3620-3623

13. Fan C, Shi Z, Pan YT, Song ZF, Zhang WJ, et al. (2014) Dual matrix-based immobilized trypsin for complementary proteolytic digestion and fast proteomics analysis with higher protein sequence coverage. Anal Chem 86: $1452-1458$

14. Lin HJ, Liu YF, Huo JR, Zhang AH, Pan YT, et al. (2013) Modified enzymelinked immunosorbent assay strategy using graphene oxide sheets and gold nanoparticles functionalized with different antibody types. Anal Chem 85: 6228-6232.

15. Jiao L, Yan HY, Xu WQ, Wu Y, Gu WL, et al. (2019) Self-assembly of allinclusive allochroic nanoparticles for the improved ELISA. Anal Chem 91(13): 8461-8465.

16. Peng HP, Huang ZN, Wu WH, Liu MK, Huang KY (2019) All inorganic mixed halide perovskite nanocrystal-graphene hybrid photodetector: from ultrahigh gain to photostability. ACS Appl. Mater. Interfaces 11(6): 24812-24819.

17. Zhu XX, Xiong SD, Zhang JQ Zhang XY, Tong X, et al. (2018) Improving paper-based ELISA performance through covalent immobilization of antibodies. Sens. Actuators B 255: 598-604.

18. Liu T, Zhang WJ, Zhang Z, Chen ML, Wang JH, et al. (2018) Sensitive western-blot analysis of azide-tagged protein post translational modifications using thermoresponsive polymer self-assembly. Anal Chem 90: 2186-2192.

ISSN: 2574-1241

DOI: 10.26717/BJSTR.2020.24.004095

Xinghe Wang. Biomed J Sci \& Tech Res

(c) (P) This work is licensed under Creative

Submission Link: https://biomedres.us/submit-manuscript.php
19. Yang H, Kong Q, Wang S, Xu J, Bian Z, et al. (2014) Hand-drawn\&written pen-on-paper electrochemiluminescence immunodevice powered by rechargeable battery for low-cost point-of-care testing. Biosens Bioelectron 61: 21-27.

20. Guo Q, Han JJ, Shan S, Liu DF, Wu SS, etal. (2016) DNA-based hybridization chain reaction and biotin-streptavidin signal amplification for sensitive detection of Escherichia coli 0157: H7 through ELISA. Biosens. Bioelectron 86: 990-995.

21. Shih CM, Chang CL, Hsu MY, Lin JY, Kuan CM, et al. (2015) Paper-based ELISA to rapidly detect Escherichia coli. Talanta 145: 2-5.

22.Zheng K, Ren J, He J (2019) Thermally responsive unimolecular nanoreactors from amphiphilic dendrimer-like copolymer prepared via anionic polymerization and cross metathesis reaction. Macromolecules 52: 6780-6791.

23. Maritschnegg E, Heinzl N, Wilson S, Deycmar S, Niebuhr M, et al. (2018) Polymer-ligand-based ELISA for robust, high-throughput, quantitative detection of p53 aggregates. Anal Chem 90(22): 13273-13279.

24. Wang K, Wang Y, Zhang W (2017) Synthesis of diblock copolymer nanoassemblies by PISA under dispersion polymerization: comparison between ATRP and RAFT. Polym Chem 8: 6407-6415.

25. Chen F, Hou SK, Li Q Fan H, Fan R, et al. (2014) Development of atom transfer radical polymer-modified gold nanoparticle-based enzymelinked immunosorbent assay (ELISA). Anal Chem 86: 10021-10024

26. Qin WJ, Song ZF, Fan C, Zhang WJ, Cai Y, et al. (2012) Trypsin immobilization on hairy polymer chains hybrid magnetic nanoparticles for ultra-fast, highly efficient proteome digestion, facile 180 labeling and absolute protein quantification. Anal. Chem. 84: 3138-3144.

27. Yang Q, Ulbricht M (2012) Novel membrane adsorbers with grafted zwitterionic polymers synthesized by surface-initiated ATRP and their salt-modulated permeability and protein binding properties Chem Mater 24: 2943-2951.

28. Fukazawa K, Nakao A, Maeda M, Ishihara K (2016) Photoreactive initiator for surface-initiated ATRP on versatile polymeric substrates. ACS Appl Mater Interfaces 8: 24994-24998.

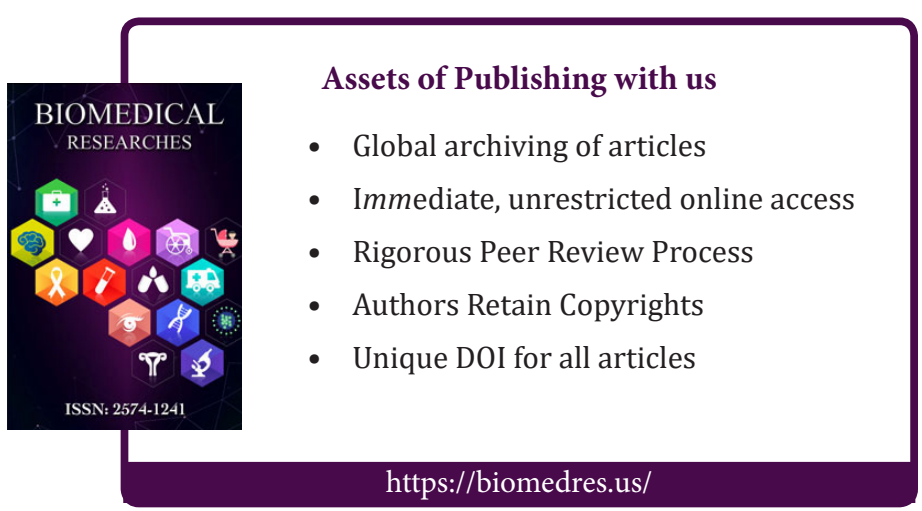

